EPJ Web of Conferences 60, 20014 (2013)

DOI: $10.1051 /$ epjconf $/ 20136020014$

(C) Owned by the authors, published by EDP Sciences, 2013

\title{
Measurement of the WW Production Cross Section in Proton-Proton Colli- sions with the ATLAS Detector
}

\author{
Jiří Hejbal ${ }^{1, a}$ on behalf of the ATLAS Collaboration. \\ ${ }^{1}$ Institute of Physics, Academy of Sciences of the Czech Republic, Praha, Czech Republic
}

\begin{abstract}
Measurements of the $\mathrm{W}^{+} \mathrm{W}^{-}$production using the leptonic decay channels are analysed using data collected by the ATLAS detector at the CERN Large Hadron Collider. The precise measurement of W+Wproduction is important for comparing to the Standard Model expectation, and also provides a detailed understanding of this process as a background in searches for new physics phenomena.
\end{abstract}

\section{Introduction}

Measurements of vector boson pair production at particle colliders provide important tests of the electroweak sector of the Standard Model (SM). Vector boson pair production at the Large Hadron Collider [1] (LHC) also represents an important source of background to Higgs boson production and to searches for physics beyond the SM. Diboson processes also provide new constraints on Triple Gauge Couplings. The latest ATLAS results on WW production are presented using data collected at centre-of-mass energy of $7 \mathrm{TeV}$.

\section{ATLAS detector}

The ATLAS detector [2] consists of an inner tracking system (inner detector, or ID) surrounded by a superconducting solenoid providing a $2 \mathrm{~T}$ magnetic field, electromagnetic and hadronic calorimeters, and a muon spectrometer (MS) incorporating three large superconducting toroid magnets arranged with an eight-fold azimuthal coil symmetry around the calorimeters. The ID consists of silicon pixel and microstrip detectors, surrounded by a transition radiation tracker. The electromagnetic calorimeter is a lead/liquid-argon (LAr) detector. Hadron calorimetry is based on two different detector technologies, with scintillator tiles or LAr as active media, and with either steel, copper, or tungsten as the absorber material. The MS comprises three layers of chambers for the trigger and for track measurements.

\section{SM WW production}

We present a measurement of $W^{+} W^{-}$inclusive and differential production cross sections in pp collisions and limits on anomalous $W W Z$ and $W W \gamma$ triple gauge couplings (TGCs) in purely leptonic decay channels $W W \rightarrow \ell v \ell^{\prime} v^{\prime}$

\footnotetext{
a e-mail: jiri.hejbal@cern.ch
}

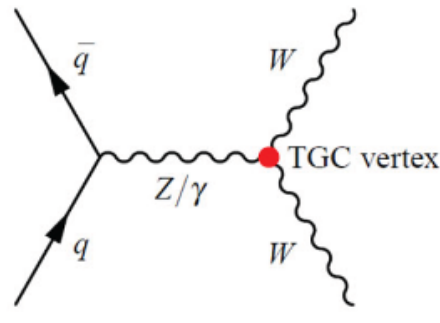

(a)

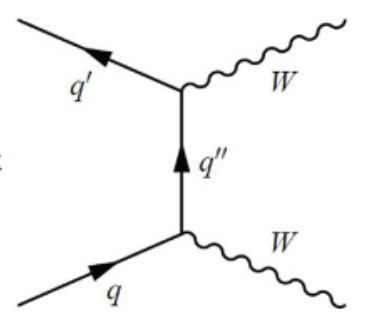

(b)
Figure 1. SM LO Feynman diagrams for $W W$ production through the $q \bar{q}$ initial state at the LHC for (a) the $s$-channel and (b) the $t$-channel [3].

with $\ell, \ell^{\prime}=e, \mu$. Processes with $\tau$ leptons decaying into electrons or muons with additional neutrinos are also included. Three final states are considered based on the lepton flavor, namely $e^{+} e^{-} E_{T}^{m i s s}, \mu^{+} \mu^{-} E_{T}^{\text {miss }}$ and $e^{ \pm} \mu^{\mp} E_{T}^{\text {miss }}$.

Leading-order (LO) Feynman diagrams for $W W$ production at the LHC include $s$-channel production with either a $Z$ boson or a virtual photon as the mediating particle or $u$ - and $t$-channel quark exchange. The $s$ - and $t$-channel diagrams are shown in Fig. 1.

Gluon-gluon fusion processes contribute a background of about 3\% to the total cross section [3]. The processes involving SM Higgs Boson production $(\approx 3 \%$ for Higgs mass of $126 \mathrm{GeV}$ ), vector boson fusion $(\mathrm{VBF})$ and double parton scattering (DPS) are not considered in estimating the contribution of background processes.

\section{Event selection}

After applyping data quality requirements and selection criteria for electrons, muons, jets and vertices [3], the events are dominated by Drell-Yan processes. To reject these background contributions, different requirements are applied to each final state. The variables used are the dilep- 

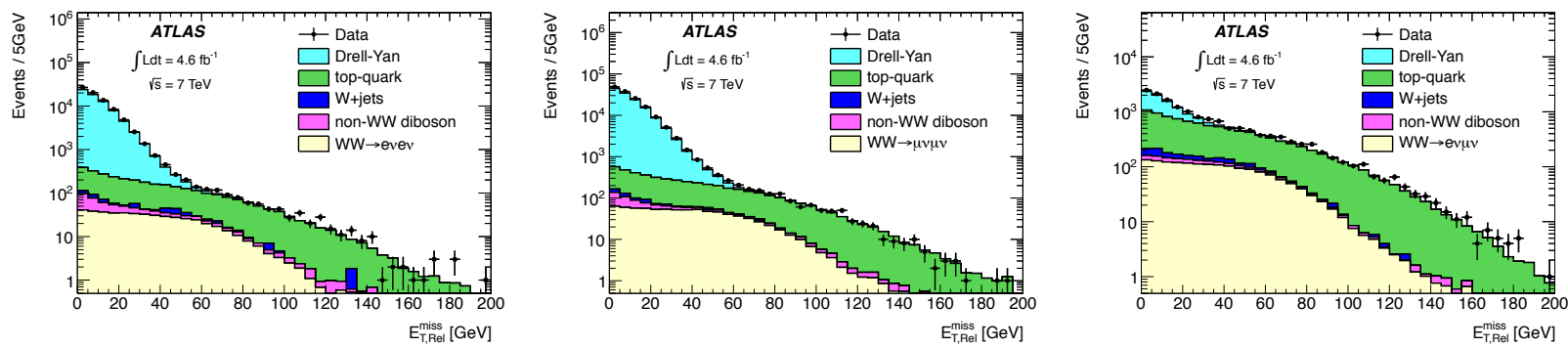

Figure 2. Comparison between data and predictions of various generators at detector level for the $E_{\mathrm{T}, \mathrm{Rel}}^{\mathrm{miss}}$ distribution before the $E_{\mathrm{T}, \mathrm{Rel}}^{\mathrm{miss}}$ cut for the $e e, \mu \mu$ and $e \mu$ channels, respectively [3].

ton invariant mass $m_{\ell \ell^{\prime}}$ and a modified missing transverse energy, $E_{\mathrm{T}, \text { Rel }}^{\text {miss }}$, definied as:

$$
E_{\mathrm{T}, \text { Rel }}^{\text {miss }}= \begin{cases}E_{\mathrm{T}}^{\text {miss }} \times \sin (\Delta \phi) & \text { if } \Delta \phi<\pi / 2 \\ E_{\mathrm{T}}^{\text {miss }} & \text { if } \Delta \phi \geq \pi / 2,\end{cases}
$$

where $\Delta \phi$ is the difference in the azimuthal angle between the $\vec{E}_{\mathrm{T}}^{\mathrm{miss}}$ and the nearest lepton or jet.

The $E_{\mathrm{T} \text {, Rel }}^{\mathrm{miss}}$ variable is designed to reject events where the apparent $E_{\mathrm{T}}^{\text {miss }}$ arises from a mismeasurement of lepton momentum or jet energy. The selection criteria applied to $m_{\ell \ell^{\prime}}$ and $E_{\mathrm{T}, \mathrm{Rel}}^{\mathrm{miss}}$ are:

- $m_{\ell \ell^{\prime}}>15,15,10 \mathrm{GeV},\left|m_{\ell \ell^{\prime}}-m_{Z}\right|>15,15,0 \mathrm{GeV}$

- $E_{\mathrm{T}, \text { Rel }}^{\mathrm{miss}}>45,45,25 \mathrm{GeV}$

for the $e e, \mu \mu$ and $e \mu$ channels, respectively. With the application of the $m_{\ell \ell^{\prime}}$ and $E_{\mathrm{T} \text {, Rel }}^{\mathrm{mis}}$ selection criteria, the remaining background events come mainly from $t \bar{t}$ and single top-quark processes. To reject this background contribution, events are vetoed if there is at least one jet candidate with $p_{\mathrm{T}}>25 \mathrm{GeV}$ and $|\eta|<4.5$. To further reduce the Drell-Yan contribution, the transverse momentum of the dilepton system, $p_{\mathrm{T}}\left(\ell \ell^{\prime}\right)$, is required to be greater than $30 \mathrm{GeV}$ for all three channels.

For illustration, Figure 2 shows comparison between data and predictions of the MC generators at detector level for the $E_{\mathrm{T}, \mathrm{Rel}}^{\mathrm{miss}}$ distribution before the $E_{\mathrm{T}, \mathrm{Rel}}^{\mathrm{miss}}$ cut is applied to the $e e, \mu \mu$ and $e \mu$ channels, respectively.

\section{Cross sections}

The $W W$ cross section is measured in the fiducial phase space and extrapolated to the total phase space. The fiducial phase space is defined at the particle level by selection criteria similar to those used at detector level and includes the jet veto as well. The fiducial cross section for the $p p \rightarrow W W+X \rightarrow \ell v \ell^{\prime} v^{\prime}+X$ process is calculated using:

$$
\sigma_{W W}^{\mathrm{fid}}=\frac{N_{\text {data }}-N_{\mathrm{bkg}}}{C_{W W} \times \mathcal{L}}
$$

where $N_{\text {data }}$ and $N_{\text {bkg }}$ are numbers of observed data events and estimated background events, respectively. $C_{W W}$ is defined as the ratio of the number of events satisfying all selection criteria at detector level to the number of events

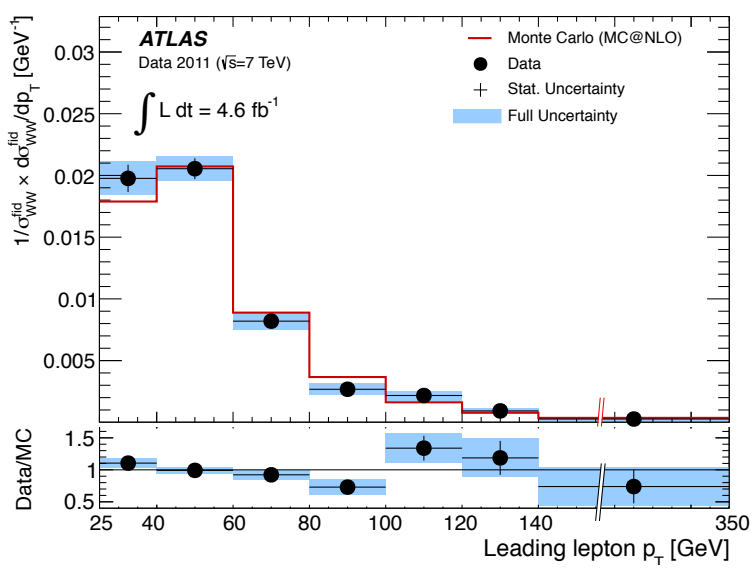

Figure 3. The normalized differential $W W$ cross section for data corrected for detector effects as a function of the leading lepton $p_{\mathrm{T}}$ compared to the SM prediction [3].

produced in the fiducial phase space and is estimated from simulations. $\mathcal{L}$ is the integrated luminosity of the data sample.

The total cross section $\sigma_{W W}$ for the inclusive $p p \rightarrow$ $W W+X$ process is calculated for each channel using:

$$
\sigma_{W W}=\frac{N_{\text {data }}-N_{\text {bkg }}}{C_{W W} \times A_{W W} \times \mathrm{BR} \times \mathcal{L}}
$$

where $A_{W W}$ represents the kinematic and geometric acceptance from the total phase space to the fiducial phase space, and BR is the branching ratio for both $W$ bosons decaying into $e v$ or $\mu v$ (including decays through $\tau$ leptons with additional neutrinos).

To obtain the normalized differential $W W$ cross section in the fiducial phase space $\left(1 / \sigma_{W W}^{\mathrm{fid}} \times d \sigma_{W W}^{\mathrm{fid}} / d p_{\mathrm{T}}\right)$, the reconstructed leading lepton $p_{\mathrm{T}}$ distribution is corrected for detector effects after the subtraction of background contamination and unfolded to the particle level. The resulting normalized fiducial cross sections extracted in bins of the leading lepton $p_{\mathrm{T}}$ together with the SM predictions is shown in Fig. 3.

\section{Signal and background yields}

The number of events selected in data and the estimated background contributions for the three individual chan- 
Table 1. Summary of observed and expected numbers of signal and background events in three individual channels and their combination [3].

\begin{tabular}{lcccc}
\hline \hline & $e e$ & $\mu \mu$ & $e \mu$ & Combined \\
\hline Data & 174 & 330 & 821 & 1325 \\
\hline$W W$ & 100 & 186 & 538 & 824 \\
\hline Top & 22 & 32 & 87 & 141 \\
$W+$ jets & 21 & 7 & 70 & 98 \\
Drell-Yan & 12 & 34 & 5 & 51 \\
Other dibosons & 13 & 21 & 44 & 78 \\
Total background & 68 & 94 & 206 & 369 \\
\hline Total expected & 169 & 280 & 744 & 1192 \\
\hline \hline
\end{tabular}

nels and the combined channel is summarized in Table 1. The expected numbers of $W W$ signal events for the individual and the combined channels are also shown. In total $1325 \ell \ell^{\prime}+E_{\mathrm{T}}^{\mathrm{miss}}$ candidates are observed in data with $824 \pm 4$ (stat) \pm 69 (syst) signal events expected from the $W W$ process and $369 \pm 31$ (stat) \pm 53 (syst) background events expected from non- $W W$ processes.

\section{Background}

The processes producing the $\ell \ell^{\prime}+E_{\mathrm{T}}^{\text {miss }}$ signature with no reconstructed jets in the final state are $\mathrm{Z}+$ jet (Drell-Yan background) when apparent $E_{\mathrm{T}}^{\text {miss }}$ is generated from the mismeasurement of the $p_{\mathrm{T}}$ of the two leptons from $Z$ boson decay; top-quark production when b-jets are not reconstructed or identified (top-quark background); $\mathrm{W}+$ jet production when a jet is misidentified as a lepton $(W+$ jets background); $\mathrm{W}+\gamma$ production with photon identified as an electron; $W+\gamma^{*}$ when one lepton is not detected; $W Z$ $\rightarrow l l l v$ where one lepton not detected; $Z Z \rightarrow l l v v$ where invariant mass is not near the Z-mass. The contribution from QCD multijet production when two jets are reconstructed as leptons is found to be negligible.

\section{Extracted TGC Limits}

The reconstructed leading lepton $p_{\mathrm{T}}$ distribution is also used to set limits on anomalous $W W Z$ and $W W \gamma$ TGCs. The Lorentz invariant Lagrangian describing the $W W Z$ and $W W \gamma$ interactions has 14 independent coupling parameters. Assuming electromagnetic gauge invariance and $\mathrm{C}$ and $\mathrm{P}$ conservation, the number of independent parameters reduces to five: $g_{1}^{Z}, \kappa_{Z}, \kappa_{\gamma}, \lambda_{Z}$ and $\lambda_{\gamma}$. Each of the couplings is usually modified by a factor depending on scale $\Lambda$ with the general form $\alpha(\hat{s})=\alpha_{0} /\left(1+\hat{s} / \Lambda^{2}\right)^{2}$, where $\alpha_{0}$ is the coupling value at low energy and $\sqrt{\hat{s}}$ is the invariant mass of the WW pair.

In the SM, the coupling parameters have the following values: $g_{1}^{Z}=\kappa_{Z}=\kappa_{\gamma}=1$ and $\lambda_{Z}=\lambda_{\gamma}=0$. Limits in the LEP scenario, where we assume $\Delta \kappa_{\gamma}=$ $\left(\cos ^{2} \theta_{W} / \sin ^{2} \theta_{W}\right)\left(\Delta g_{1}^{Z}-\Delta \kappa_{Z}\right)$, and $\lambda_{Z}=\lambda_{\gamma}$ are compared with limits obtained from the CMS [4], CDF [5], DØ [5] and LEP [6] experiments in Fig. 4.

Table 2 shows expected and observed $95 \%$ confidence level limits on anomalous $W W Z$ and $W W \gamma$ couplings in

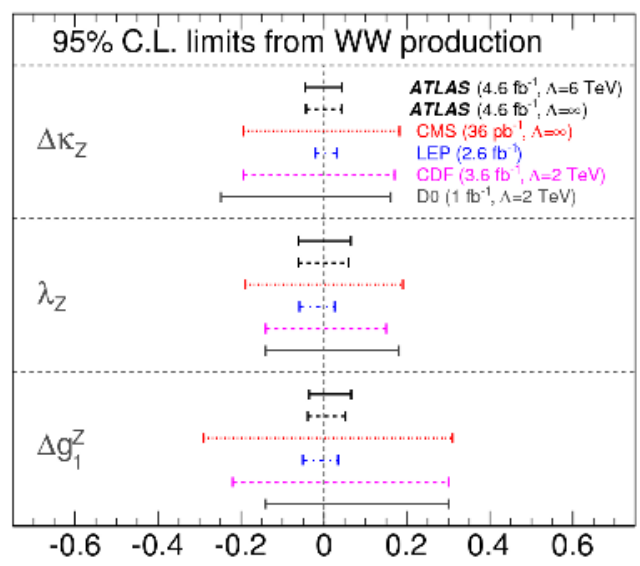

Figure 4. Comparison of anomalous TGC limits from ATLAS, CMS, CDF, DØ and LEP experiments for the LEP scenario [3].

Table 2. The expected and observed limits on anomalous TGCs in Equal Couplings scenario for QCD scale $\Lambda=6 \mathrm{TeV}$ [3].

\begin{tabular}{lcc}
\hline \hline Parameter & Expected & Observed \\
\hline$\Delta \kappa_{Z}$ & {$[-0.058,0.089]$} & {$[-0.061,0.093]$} \\
$\lambda_{Z}=\lambda_{\gamma}$ & {$[-0.060,0.062]$} & {$[-0.062,0.065]$} \\
\hline \hline
\end{tabular}

the Equal Couplings scenario for QCD scale $\Lambda=6 \mathrm{TeV}$. In this scenario we assume that the $W W Z$ and $W W \gamma$ couplings are equal $\left(\Delta \kappa_{Z}=\Delta \kappa_{\gamma}, \lambda_{Z}=\lambda_{\gamma}\right.$, and $\left.g_{1}^{Z}=1\right)$.

\section{Summary and results}

The WW inclusive production cross section in protonproton collisions at $\sqrt{s}=7 \mathrm{TeV}$ is measured using $4.6 \mathrm{fb}^{-1}$ of data collected with the ATLAS detector at the LHC. In total 1325 candidates are selected with an estimated background of $369 \pm 61$ events channels into $e e, \mu \mu$ and $e \mu$ final states.

The combined production cross section $\sigma(p p \rightarrow$ $W W+X) 51.9 \pm 2.0$ (stat) \pm 3.9 (syst) \pm 2.0 (lumi) pb, compatible with the SM NLO prediction of $44.7_{-1.9}^{+2.1} \mathrm{pb}$.

\section{References}

[1] L. Evans and P. Bryant 2008 JINST 3 S08001.

[2] ATLAS Collaboration, 2008 JINST 3 S08003.

[3] ATLAS Collaboration, Phys. Rev. D 87, 112001 (2013).

[4] CMS Collaboration, Phys. Lett. B 699, 25 (2011).

[5] CDF Collaboration, Phys. Rev. Lett. 104, 201801 (2010); D0 Collaboration, Phys. Rev. Lett. 103, 191801 (2009); ibid., Phys. Rev. D 80, 053012 (2009).

[6] ALEPH Collaboration, Phys. Lett. B 614, 7 (2005); DELPHI Collaboration, Eur. Phys. J. C 54, 345 (2008); L3 Collaboration, Phys. Lett. B 586, 151 (2004); OPAL Collaboration, Eur. Phys. J. C 33, 463 (2004). 